\title{
Diagnostic importance of Ultrasound-Guided Fine Needle Aspiration in Diagnosing Hepatic Lesions among Sudanese Patients 2015
}

\author{
Ali Mahmoud Mohammed Edris ${ }^{1 *}$, Imtithal Mohamed Ali², Shaimaa Bushra ${ }^{3}$ \\ Bakeit, Mohamed Abashar ${ }^{1}$, Emmanuel Edwar Siddig ${ }^{1}$
}

\begin{abstract}
Background: Liver cytology is indicated and requested for evaluating hepatic masses in symptomatic or serendipitous cryptic discovered lesions. Objective: To determine the cytomorphological patterns of hepatic lesions identified among a group of Sudanese patients. Materials and Methods: This is an analytical descriptive hospital-based study included 165 patients undergoing ultrasound-guided fine needle is an aspiration cytology (FNAC)for hepatic lesions, at Al-Amal Military Hospital \& Khartoum Teaching Hospital in Khartoum, Sudan. Clinical data were reviewed. Air dried Diff Quick stained smears were grouped into unsatisfactory samples, benign lesions, and malignant neoplasms. Results: Our population were consisted of $35(21.2 \%)$ females and $130(78.8 \%)$ males, with a male to female ratio $3.7: 1$ and an age ranged between 47 to 80 , and a mean age $57 \pm 7$. Of 165 cases, $57(34.5 \%)$ were benign, no atypia were noticed, $101(61.2 \%)$ were malignant. Most investigated patients were found to have metastatic lesions. Conclusion: FNAC is a useful tool for investigating hepatic lesions.
\end{abstract}

Keywords: Fine needle aspiration - hepatic lesions - metastatic cancers

Asian Pac J Cancer Prev, 17 (2), 553-555

\section{Introduction}

The main indication for cytologic study of the liver is the evaluation of mass-forming lesions, either symptomatic or incidentally discovered. Fortunately, recent major significant progress in cutting-edge imaging methods is shifting the spectrum of cytologic diagnostics toward smaller lesions, which are very often challenging (Wee, 2011). The convenience of relatively easy fine needle aspiration (FNA) procedures with high diagnostic accuracy and low risk for complications has made cytologic examination of primary and metastatic neoplasms in the liver generally well accepted as a first line diagnostic modality (Chhieng, 2004; Kuo et al., 2004; Wee, 2005; Pupulim et al., 2008; Jenssen and Dietrich, 2009). Furthermore, for most patients, treatment (including major surgery or chemotherapy) may be safely instituted based on the diagnosis of a malignant neoplasm in FNA, without obligatory histologic confirmation. In cases of metastatic disease, the availability of diagnostic cytologic material to determine predictive markers becomes progressively more significant.

Liver FNAC is a safe technique with a low rate of serious complications, mostly bleeding or infection
(Wang et al., 2008). Absolute contraindications for liver FNAC are markedly abnormal coagulation parameters, and relative contraindications include the location of focal lesions in the liver parenchyma that cannot be safely accessed because they are close to large vascular structures. Liver FNAC is almost always guided by either ultrasound or computed tomography (CT) (Crowe et al., 2006). Remarkably Ultrasound offers the advantage of real-time visualization of the needle tip. Most liver FNAs are performed percutaneous. Depending upon the topographical relationship of the lesion in question, an endoscopic transesophageal approach may be an option in selected cases for an endoscopic ultrasound (EUS)-FNA of the liver. Importantly, recent studies have reported good sensitivity of FNA for liver malignancy, ranging between 67 to $100 \%$, and specificity of $100 \%$ or close to it. Falsepositive diagnoses are rare and positive predictive values approach $100 \%$ (Jenssen and Dietrich, 2009; Maheshwari et al., 2010; Wee, 2011). Thus, overdiagnosing malignancy is probably less of a problem than underdiagnosing it. Reasons for false negatives include paucity of tumor cells and failure to recognize tumor cells with subtle malignant features. 


\section{Materials and Methods}

This is an analytical descriptive hospital based study in which we reviewed records of all patients referred to Al-Amal Military Hospital \& Khartoum Teaching Hospital at Khartoum state - Sudan for evaluating Hepatic lesions with normal prothrombin time were subjected to USG - FNA, From January 2012 till December 2014. All clinical data were retrieved from the records. The cytological material was obtained using 20 or 22-gauge, 90-mm spinal needle which was introduced into the lesion under ultrasound guidance. The smears were stained by May-Grunwald-Giemsa, Papanicolaou, hematoxylin and eosin (H and E) stains. Diagnosis was established by two well expertise cytopathologists and the diagnosed smears were categorized into inadequate or nondiagnostic if insufficient cellular material was present and no evidence of cellular atypia was found, Normal, Benign, suspicious for malignancy and malignant.

Smears from 165 patients were viewed, the main cytomorphological features used for categorization included: cellularity of smears, cellular architectures (Branching, trabecular formation, three dimensional, monolayer sheets), and nuclear features, necrosis in the background and presence or absence of bile duct epithelial. Data were analyzed by using a computer based Statistical Package for the Social Sciences SPSS version 16. Pearson Chi Square Test was used to test association between variables. Ethical considerations: ethical approval was obtained from ethics committee at Faculty of Medical Laboratory Science, University of Khartoum. Both written and verbal consent were obtained from each individuals enrolled in this study.

\section{Results}

A total of 165 patients were examined at cytology Clinic between January 2012 till December 2014. Our study population were comprised of 35 (21.2\%) females, $130(78.8 \%)$ were males, with male to female ratio 3.7 : 1 , with an age ranged between 47 to 80 , and a mean age $57 \pm 7$. All the aspirates were satisfactory for interpretation with the exception of $7(4.2 \%)$ classified as inadequate for interpretation due to low cellularity. The result was classified into 3 cytological diagnoses: 57 (34.5\%) were benign, $101(61.2 \%)$ were malignant lesions.

Regarding the benign lesions, 28 (49.1\%) were fatty metamorphosis, 16 (28\%) were hepatic abscess, 9 (15.7\%) were alcoholic hepatitis, and 4 (7\%) were hepatocellular adenomas. With respect to the malignant lesions, 20 (19.8\%) were found to be hepatocellular carcinoma . Moreover, the distribution of hepatic lesions among studied population represents about $81(80.1 \%)$.

Regarding age group, the peak age group for hepatic lesions was 56 to 70 years comprising $118(71.51 \%)$ of the total populations, followed by the age group 40 to55, $(21.81 \%)$ patients. It is clear that the risk of cancer increased from the 4 th decades of life with the peak at $5^{\text {th }}$ till $7^{\text {th }}$ decades the result showed to be statistically significant (P value < 0.003 ) (Table.1.). Our results also shown that there is no statistical significance between gender and having hepatic lesions.

\section{Discussion}

The liver is a common site for various non-neoplastic and neoplastic lesions such as primary liver tumors (benign or malignant), metastatic deposits, congenital and acquired cysts, abscesses and granulomas. The appropriate clinical management of liver lesions depended upon accurate diagnosis which is a dilemma (Kuo et al., 2004; Leiman, 2005; Mohmmed et al., 2012). Liver cancer is the sixth most common cancer worldwide (Lenhard et al., 2001; El-Serag, 2002; Ashwin et al., 2006), accounting for $5.7 \%$ of the overall incident cases of cancer. There is wide geographic variability in incidence with a majority of the cases occurring in developing countries $(82 \%, 366,000$ new cases estimated in males in 2002, 147,000 in women) compared with developed countries $(74,000$ new cases in men and 36,000 women) (Pakin et al., 2005). Surprisingly, in fact, liver cancer is the third most common cancer in developing countries among men after lung and stomach cancer. It is also between two and eight times more common in men than in women. In Sudan, cancer is the third leading cause of death-after malaria and pneumoniawhich is accounting for $5 \%$ of all deaths. Liver cancer accounts for about 7.5\% of all cancers (Hamad, 2006).

Differentiation of primary liver cancer from metastatic tumors and early detection of hepatocellular carcinoma are of special importance in our clinical practice in view of the relatively favorable prognosis with resection. With the development of sophisticated imaging techniques, early detection of liver cancer is now possible. In the senior author's series, the smallest cancerous lesion of the liver diagnosed by guided fine-needle aspiration (FNA) biopsy was $7 \mathrm{~mm}$. Cell block and immunohistochemistry further enhance diagnostic accuracy in the typing of hepatic cancers. The sensitivity of guided FNA for a diagnosis of hepatic malignancy ranges from 90 to $96 \%$, with a specificity of $100 \%$, similarto thick-needle core biopsies (Pagani, 1983; Dusenbery et al., 1995).

Overall in our study, 57 (34.5\%) were benign, 101 $(61.2 \%)$ were malignant lesions and about $80 \%$ of malignant lesions were metastatic or non primary, this result is in agreement with that of Tao et al., 1980, after reviewing of 1691 cases of aspiration biopsy of the liver, 1217 (72\%) were metastatic cancers (Tao et al., 1980). Some other workers have reported metastatic liver malignancy as high as 90\% (Orell et al., 1992). In our present study the most common cancer metastasized to the liver were Colon cancer (39.6\%), followed by Lung

Table 1. Association between Age Group and Cytological Findings

\begin{tabular}{lcccr}
\hline Age group & Inadequate & $\begin{array}{c}\text { Diagnosis } \\
\text { Benign }\end{array}$ & Malignant & Total \\
\hline $40-55$ years & 1 & 22 & 13 & 36 \\
$56-70$ years & 5 & 34 & 79 & 118 \\
71 and more & 1 & 1 & 9 & 11 \\
Total & 7 & 57 & 101 & 165 \\
\hline
\end{tabular}

(P value $<0.003)$ 
Diagnostic Importance of Ultrasound-Guided FNCA in Diagnosing of Hepatic Lesions among Sudanese Patients in 2015

(21.7\%), Non Hodgkins lymphoma and adenocarcinoma of gall bladder $(7.9 \%)$ for each this results in agreement with (Abdul- Raheem and Elfaki, 2013). Furthermore our results shown similarity to that conducted by Mustafa et al., 2014; after examining 400 liver smears, they show that the most common malignancy were adenocarcinoma, with colonic adenocarcinoma is the most common metastasis to the liver (Mustafa et al., 2014). Furthermore, Pinto et al., 1988, observed two cases of metastatic renal cell carcinoma, in our present study 3 cases of renal cell carcinoma metastasized to liver were notice.

According to our results, it shown that there is a significant association between the age of the patient and developing cancer $(\mathrm{P}$ value $<0.003)$, with most cancer associated with age group between 56 to 70, interestingly, According to American Cancer Society, The average age at diagnosis of liver cancer is 63. More than $95 \%$ of people diagnosed with liver cancer are 45 years of age or older. About $3 \%$ are between 35 and 44 years of age and about $2 \%$ are younger than 35 (American Cancer Society, 2014).

The most diagnostic dilemma we encountered in the liver cytopathology is the recognition of poorly differentiated hepatocellular carcinoma, however this type of malignancy is easily recognized as malignant but too poorly differentiated to be recognized as hepatic origin based on cytomorphology alone (Tao et al., 1984; Yang and Tao, 2007). Fine needle aspiration reveals few cohesive cells clusters, these cells appear bizarre and contain large round, to oval nuclei with prominent nucleoli, binucleation and eccentric to central located nucleus were notice, in this situation the correlation of cytological finding with clinical and radiological finding is clue for the final diagnosis, as well as using immunohistochemistry to exclude the possibility of metastasis aid in the final diagnosis. Interestingly, Mustafa et al., 2014, agree with our finding in that the major diagnostic dilemma in differentiating between poorly differentiating HCC from Metastatic adenocarcinoma.

Fine needle aspiration cytology is gaining popularity as a means of diagnosing hepatic lesions, and it proved to be superior to core needle or open biopsy in term of cost, procedures, associated morbidity and early diagnosis (Mustafa et al.,2014).

In conclusion, FNAC of the liver is a simple, safe, accurate, economicalscreening test that can be used to identify the vastmajority of neoplasms of a primary or metastatic nature.

\section{Acknowledgements}

We would like to offer our great appreciation to personnel at department of radiology and the medical laboratory at Al-Amal Military hospital.

\section{References}

Abdul- Raheem E, and Elfaki E (2013). The role of fine needle aspiration cytology in the diagnosis of deep-seated lesions in sudanese patients. International Journal of Health Sciences \& Research, 3, 10 .

Ashwin A, Veena G and Kia Saeian (2006), Epidemiology of primary and secondary liver cancers. Semin Intervent Radiol, 23, 47-63.

Crowe D, Eloubeidi M, Chhieng D, et al (2006). Fineneedle aspiration biopsy of hepatic lesions: computerizedtomographic-guided versus endoscopic ultrasound-guided FNA. Cancer, 108, 180-5.

Chhieng D (2004). Fine needle aspiration biopsy of liver - an update. World J Surg Oncol, $2,5$.

Dusenbery D, Ferris J, Thaete F, et al (1995). Percutaneous ultrasound-guided needle biopsy of hepatic mass lesions using a Cytohistological approach: comparison of two needle types. Am J Clin Pathol, 104, 583-7.

El-Serag H (2002). Hepatocellular carcinoma: an epidemiologic view. J Clin Gastroenterol, 35, 72-8.

Hamad H (2006). Cancer initiatives in Sudan. Ann Oncol, 8, 32-6.

Jenssen C, Dietrich C (2009). Endoscopic ultrasoundguided fi ne-needle aspiration biopsy and trucut biopsy in gastroenterology - an overview. Best Pract Res Clin Gastroenterol, 23, 743-59.

Kuo FY, Chen WJ, Lu SN, et al (2004). Fine needle aspiration cytodiagnosis of liver tumors. Acta Cytol, 48,142-8.

Leiman G (2005). Liver and Spleen. In: Orell SR, Sterret GF, Whitaker D, editors. Fine needle aspiration cytology. 4th ed. New Delhi: Churchill Livingstone, pp. 293-316.

Lenhard R, Osteen R, Gansler T (2001). Clinical Oncology. Atlanta, GA: Blackwell Publishing, Inc.

Maheshwari A, Kantsevoy S, Jagannath S, et al (2010). Endoscopic ultrasound and fine-needle aspiration for the diagnosis of hepatocellular carcinoma. Clin Liver Dis, 14, 325-32.

Mustafa B, Shushruta B, Manisha K, et al (2014). Fine needle aspiration cytology of lesions of liver and gallbladder: An analysis of 400 consecutive aspirations, J Cytol, 31, 20-4.

Orell S, Sterrett G, Walters M, et al (1992). Manual and Atlas of Fine Needle Aspiration Cytology.2nd ed. Hong Kong: Churchill Livingstone; Retroperitoneum, liver and spleen; pp. 217-66.

Pagani J (1983). Biopsy of focal hepatic lesions.Comparison of 18 and 22 gauge needles. Radiology, 147, 673-5.

Parkin D, Bray F, Ferlay J, et al (2005). Global cancer statistics, 2002. CA Cancer J Clin, 55, 74-108.

Pinto M, Avila N, Heller C, et al (1988). Fine needle aspiration of the liver. Acta Cytol, 32, 15-21.

Pupulim L, Felce-Dachez M, Paradis V, et al (2008). Algorithm for immediate cytologic diagnosis of hepatic tumors. AJR Am J Roentgenol, 190, 208-12.

Tao L, Pearson F, Delarue N, et al (1980). Percutaneous fineneedle aspiration biopsy. 1. Its value to clinical-practice. Cancer, 45, 1480-5.

Tao L, Ho C, McLoughlin M, et al (1984). Cytologic diagnosis of hepatocellular carcinoma by fine-needle aspiration biopsy. Cancer, 53, 547-52.

Wang P, Meng Z, Chen Z, et al (2008). Diagnostic value and complications of fi ne needle aspiration for primary liver cancer and its influence on the treatment outcome-a study based on3011 patients in China. Eur J Surg Oncol, 34, 541-6.

Wee A (2005). Fine needle aspiration biopsy of the liver: algorithmic approach and current issues in the diagnosis of hepatocellular carcinoma. Cyto journal, $2,7$.

Wee A (2011). Fine needle aspiration biopsy of hepatocellular carcinoma and hepatocellular nodular lesions: role, controversies and approach to diagnosis. Cytopathology, 22:287-305.

Yang G, Tao L (2007). Trans abdominal Fine-Needle Aspiration Biopsy. A color atlas and monograph.2nd edn.hackensackand singapore world scientific. 